\title{
Multimodal gas detection using molecular materials
}

\author{
M. Bouvet ${ }^{1}$, J.-M. Suisse ${ }^{1}$, T. Sizun ${ }^{1}$, A. Kumar ${ }^{1}$, G. Barochi ${ }^{1,2}$, B. De Fonseca ${ }^{1,2}$, J. Rossignol', \\ ${ }^{1}$ Institut de Chimie Moléculaire de l'Université de Bourgogne (ICMUB), Université de Bourgogne, \\ UMR CNRS 6302, 9 avenue A. Savary, F-21078 Dijon, France, \\ marcel.bouvet@u-bourgogne.fr \\ ${ }^{2}$ Laboratoire Interdisciplinaire Carnot de Bourgogne, Université de Bourgogne, \\ UMR CNRS 6303, 9 avenue A. Savary, F-21078 Dijon, France
}

\begin{abstract}
:
In the field of sensors, molecular materials present interesting and potentially valuable features as sensing elements for real gas sensor applications. We review the different types of conductimetric transducers. Among them, resistors have been historically the most commonly exploited way for the detection and quantification of gas pollutants. Herein, we focus on new transducers, either based on the well-known OFETs, or on transducers combining two molecular materials, namely the $p-n$ junctions and the brand new Molecular Semiconductor - Doped Insulator (MSDI) heterojunctions. We also show how a molecular material-based microwave transducer can be used to detect ammonia. The adsorption of a target gas on a material induces a change in several physical parameters, such as the dielectric constant, the work function or the conductivity. The use of different transducers sensitive to the variation of these parameters appears to be a relevant methodology worthy of investigation.
\end{abstract}

Keywords: molecular material, conductimetric transducer, microwave transducer, gas sensor, ozone, ammonia.

\section{Introduction}

Pollution, indoor or outdoor, inside buildings or due to motor vehicles, or related to specific industrial wastes, constitutes a topic of increasing interest. Among pollutants, ozone $\left(\mathrm{O}_{3}\right)$ and nitrogen dioxide $\left(\mathrm{NO}_{2}\right)$ must be measured in the ppb range in big cities, while olfactive pollutants (amines and sulfur derivatives) have to be detected in the ppm range [1]. Though no relation should be made between the presence of an odor and toxicity, since in many cases perception occurs on levels quite below the exposure limit, the perceived olfactory pollution can be quite real to the locals, and subjected to wind orientation and variations. The case of ammonia $\left(\mathrm{NH}_{3}\right)$, an olfactory pollutant, must be considered in a particular way because it is involved in many industrial applications [2]. Indeed, liquid ammonia is used as cryogenic fluid in particular in the cold rooms and more generally in food conservation, as a replacement of fluorocarbons. Any leak can prove to be detrimental for the workers carrying out their tasks inside or in the vicinity of these installations. $\mathrm{NH}_{3}$ is also produced on industrial breeding sites as a product of the natural transformation of animal excrements. The control of its concentration will make it possible to optimize ventilation of the buildings and ensure the good health of both animals and people. In addition, in the industry of manures $\mathrm{NH}_{3}$ concentration must be controlled and kept below the alarm threshold of 50 ppm in the air.

The sensors available on the market are mainly of two types [1]. The first are conductimetric sensors, generally using non stoechiometric metal oxide like $\mathrm{SnO}_{2-x}$, which present a long life span, can operate from ppm to percent ranges and are available at low costs. But their main drawback is that they require high working temperature, usually in the $300-500^{\circ} \mathrm{C}$ range, and moreover are not selective. The other kind of commercially available ammonia sensors, of the electrochemical type, is sensitive in the ppm range. Those are generally selective but are also much more expensive.

Among all the physical parameters modified by the adsorption of gases, the most involved in molecular material - based gas sensors is the work function [3]. Modulation of the work function of electronic materials by interaction with gases and vapors is without a doubt a viable and well-defined sensing principle [4]. It 
is used in equilibrium potentiometric sensors, such as the macroscopic Kelvin probe and in its solid-state miniature counterpart, the field-effect transistor, used as chemical sensors (ChemFET). However, because the workfunction is such a fundamental property of electronic materials it is also directly involved in other kind of chemical sensors, namely junctions [5] and various forms of chemiresistors [6]. In these sensors, the response is obtained while current is passing through the sensing material. Elsewhere, the microwave motion depends not only on the dielectric constant of a material but also on its conduction. This is at the origin of the development of microwave transducers for chemical sensing $[7,8]$.

\section{Conductimetric transductions}

Whereas organic FETs [9] and diodes [10] have been prepared after their inorganic counterparts, we designed and characterized a new molecular material-based device (Fig. 1). Molecular Semiconductor - Doped Insulator (MSDI) Heterojunctions were built combining a molecular semiconductor (MS) and a dopedinsulator (DI) [11]. MS must be more conductive than the sub-layer to take advantage of the heterojunction. Up until now, the intrinsic molecular semiconductor [12], namely the lutetium bisphthalocyanine $\left(\mathrm{LuPC}_{2}\right)$ and lanthanide triple-decker complexes have been used as MS [13]. DI can be p-type materials, as non-substituted Pc (ex.: CuPc), or sexithiophenes, or n-type-materials, as fluorinated $\mathrm{Pc}$ (ex:: $\mathrm{Cu}\left(\mathrm{F}_{16} \mathrm{Pc}\right)$ ) or perylene derivatives [14]. MSDIs have been used as new transducers for gas chemosensing (Fig. 2). pType materials lead to MSDls that exhibit a positive response to ozone and a negative response to ammonia, whereas MSDIs prepared from n-type materials exhibit a positive response to ammonia and negative response to ozone. The remarkable point is that the only material in contact with the analytes is in all cases the very same semiconductor.

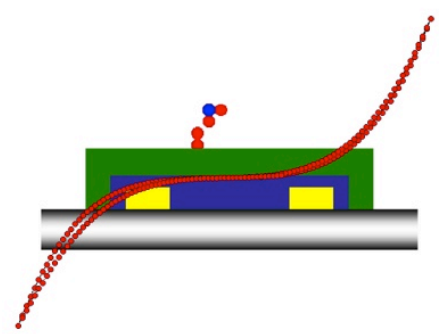

Fig. 2. Scheme of a MSDI heterojunction, and of a typical I(V) characteristics [11].

The variation of the work function is always accompanied by a change in the density of the charge carriers, so, it must be taken into account in the present sensors. Thus, gas sensor tests were performed to check the potentialities of hybrid films made from cellulose and hydroxygallium phthalocyanine (HOGaPc) [15]. In particular, the synergetic behavior between the film-forming materials allows a fast and sensible change in surface potential, measured using the Kelvin-Zisman vibrating capacitor probe, after cyclic exposures to ozone $\left(\mathrm{O}_{3}, 100 \mathrm{ppb}\right)$ and nitrogen (Fig. 3). It was shown that the hybrid film acts not only as an active layer in detecting the oxidant atmosphere with higher sensitivity and stability, but exhibits also a fast recovering of the initial properties, compared to pure phthalocyanine. The conductivity of cobalt phthalocyanine (CoPc) resistors was measured under a flow of ammonia and ozone $\left(\mathrm{NH}_{3}\right.$ and $\left.\mathrm{O}_{3}\right)$, during exposure/recovery cycles. It appears that the relative responses are linearly related to the concentration, in the 20-200 ppb range for $\mathrm{O}_{3}$ and in the 20-200 ppm range for $\mathrm{NH}_{3}$ (Fig. 4) [19].

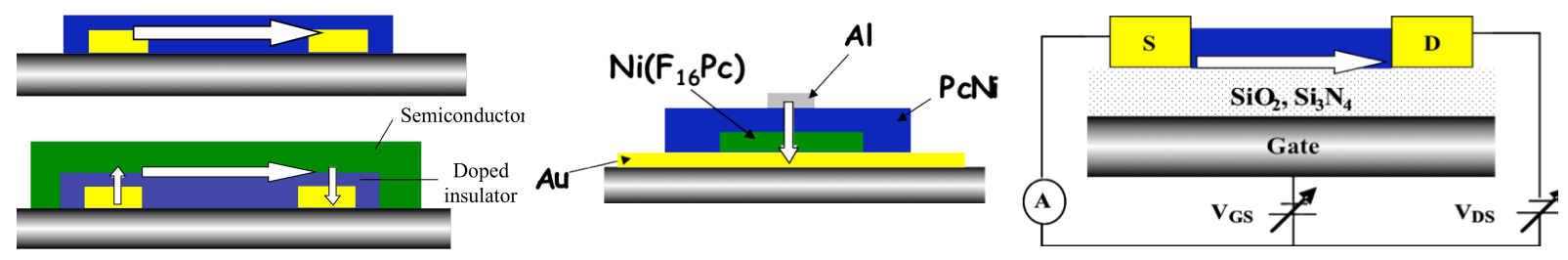

Fig. 1. Schemes of conductimetric sensors; left: resistor [1] and MSDI [11], middle: p-n junction [10]; right: FET [16-18]; the white arrows indicate the main conduction pathways 


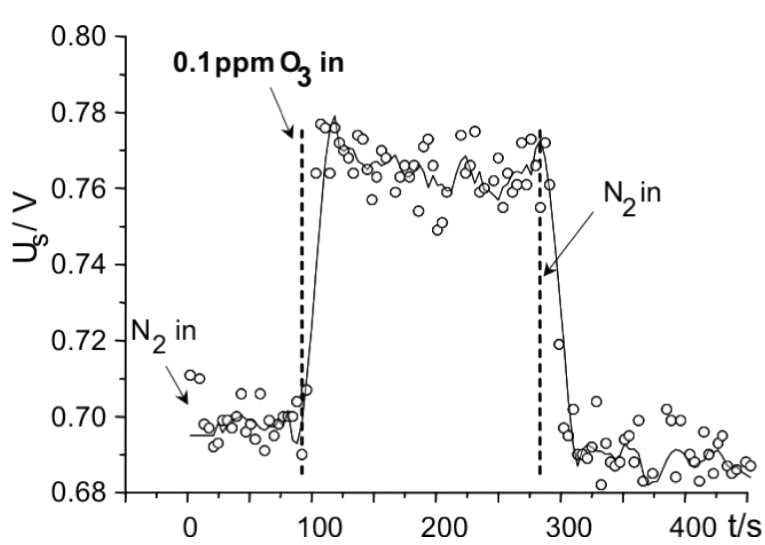

Fig. 3. Transient response of the surface potential after the alternate exposure from inert $\mathrm{N}_{2}$ to an electron-accepting agent as $\mathrm{O}_{3}(100$ ppb) measured using the Kelvin probe; a) in a 1-HOGaPc/ITO film; b) in a 1-HOGaPc/CELLR/ITO hybrid film [15].

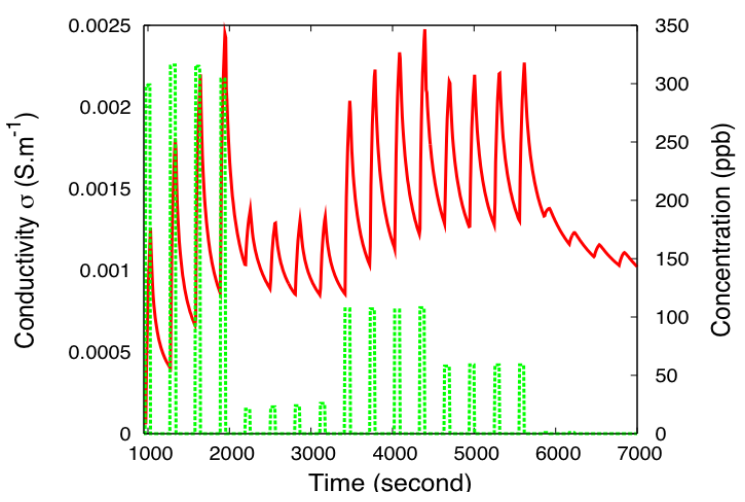

Fig. 4. Conductivity variation as a function of time of a CoPc resistor exposed to different ozone concentrations for $1 \mathrm{~min}$ periods spaced by a $4 \mathrm{~min}$ static rest period. The ozone concentration at each cycle is indicated [19].

\section{New microwave transducers}

We developed a novel approach in gas detection by using an original method of microwave transduction. Microwaves are radio waves with wavelengths ranging from as long as one meter to as short as one millimeter, or equivalently, with frequencies between $300 \mathrm{MHz}(0.3 \mathrm{GHz})$ and $300 \mathrm{GHz}$. The design of the sensor includes a coplanar grounded wave guide combined with a gas sensing molecular material [20]. The sensing material can play the role of substrate or can be deposited as a thin layer on a microstrip structure similar to those used in electronics (Inset of Fig. 5). Submitted to an electromagnetic excitation in microwave energies, the sensor response in the presence of a gas results in a specific modification of the reflected wave (real and imaginary parts). The response was defined as the ratio of the reflected wave on the incident wave. In the present investigation, the sensing material was CoPc deposited by vacuum evaporation. We showed how, in the presence of ammonia, the reflected wave is related to its concentration. Both the imaginary part and the real parts of the reflected coefficient vary with the ammonia concentration. The plot of the imaginary part as a function of the real part, at $6.8 \mathrm{GHz}$, shows domains that are related to the ammonia concentration (Fig. 5). All the measurements were carried out at room temperature. The effect of ammonia results not only from a modification of the dielectric constant of the sensing layer, but also from the variation of its conductivity. Thus, the microwave transduction is a complementary transduction mode compared to conductimetric transduction.

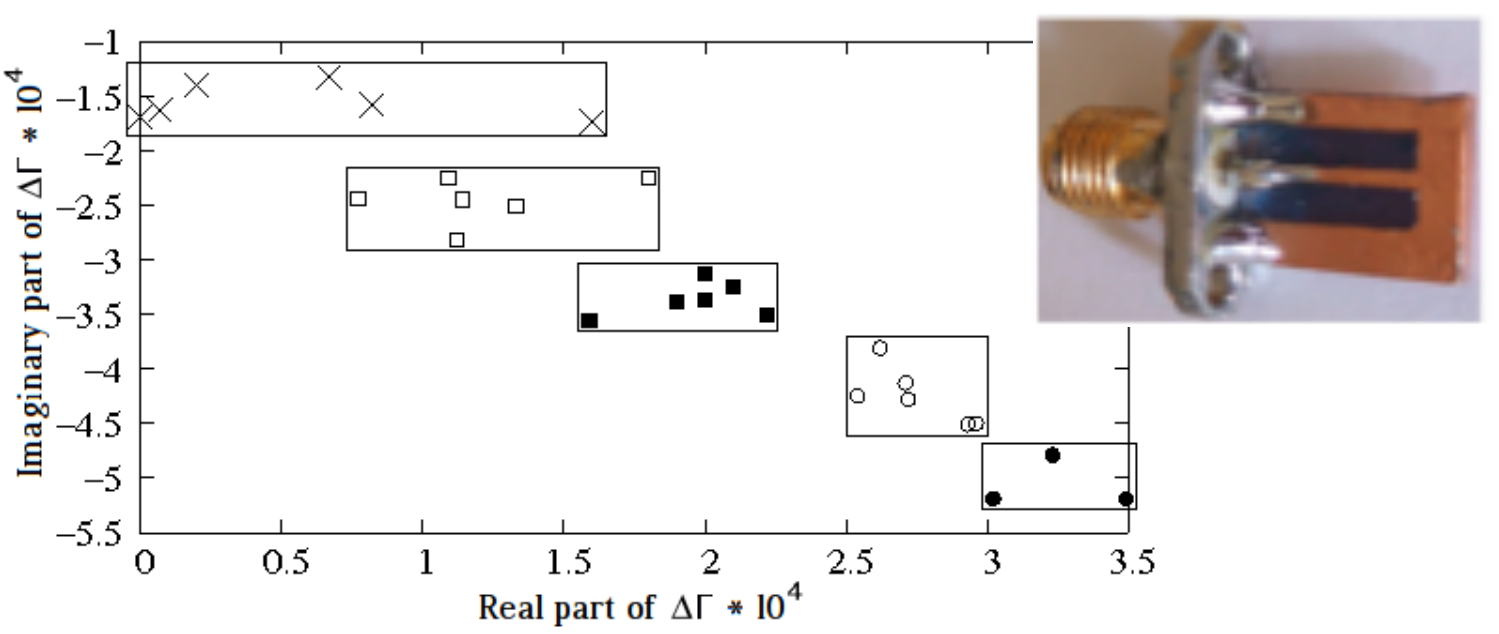

Fig. 5. Representation of the imaginary part of the reflected coefficient as a function of the real part, at $6.8 \mathrm{GHz}$; the $\mathrm{NH}_{3}$ concentration varies by steps of $100 \mathrm{ppm}$, from $100 \mathrm{ppm}$ at the upper left domain to $500 \mathrm{ppm}$ for the lower right domain. Inset: Picture of the microstrip structure [20]. 


\section{Conclusion}

Given the fact that conductimetric and microwave transduction modes are sensitive to various physical parameters, we are currently working on developing of a multimodal sensor by combining these two complementary techniques.

\section{Acknowledgements}

The authors acknowledge the Conseil Régional de Bourgogne for funding through the program PARI SMT 08 IME - Région Bourgogne. Financial support from the European Union and the Conseil Régional de Bourgogne through the FABER program is gratefully acknowledged. The authors would like to acknowledge the Agence Nationale de la Recherche (A. N. R. France) for fundings within the CAPBTX project (grant number ANR-10-BLAN-917-02).

\section{References}

[1] M. Bouvet, A. Pauly, Molecular Semiconductor Based Gas Sensors in The Encyclopedia of Sensors, edited by C.A. Grimes, E.C. Dickey, M. V. Pishko, American Scientific Publishers, vol 6, 227-270 (2006).

[2] B. Timmer, W. Olthuis, A. van den Berg, Ammonia sensors and their applications-a review Sensors and Actuators B, 107, 666-677 (2005); doi: 10.1016/j.snb.2004.11.054

[3] J. Janata, M. Josowicz, Organic semiconductors in potentiometric gas sensors, Solid State Electrochemistry 13:41-49 45 (2009); doi: $10.1007 / \mathrm{s} 10008-008-0597-0$

[4] J. Janata, Principles of chemical sensors, Plenum, New York, 1989.

[5] K. Potje-Kamloth, Semiconductor Junction Gas Sensors, Chemical Reviews 108:367-399, (2008); doi: $10.1021 / \mathrm{cr} 0681086$

[6] G. Guillaud, J. Simon, J.-P. Germain, Metallophthalocyanines: Gas sensors, resistors and field effect transistors, Coordination Chemistry Reviews 178-180, 14331484 (1998); doi: 10.1016/S0010-8545(98)00177-5

[7] J. Jouhannaud, J. Rossignol, D. Stuerga, Metal oxide-based gas sensor and microwave broadband measurements: an innovative approach to gas sensing, J. Phys.: Conf. Ser. 76, 012043 (2007); doi:10.1088/1742-6596/76/1/012043

[8] J. Rossignol, D. Stuerga, J. Jouhannaud, Broadband microwave gas sensor: A coaxial design, Microwave Opt Technol Lett 52(8), 17391741 (2010); doi: 10.1002/mop.25359

[9] G. Guillaud, M. Al Sadoun, M. Maitrot, J. Simon, M. Bouvet, Field-effect transistor based on intrinsic molecular semiconductors, Chemical Physics Letters 167, 503-506 (1990); doi: 10.1016/0009-2614(90)85459-P
[10] I. Muzikante, V. Parra, R. Dobulans, E. Fonavs, J. Latvels, M. Bouvet, A novel gas sensor transducer based on phthalocyanine heterojunction devices, Sensors, 7, 2984-2996 (2007); doi:10.3390/s7112984

[11] V. Parra, J. Brunet, A. Pauly, M. Bouvet, Molecular semiconductor - doped insulator (MSDI) heterojunctions, an alternative transducer for gas chemosensing, Analyst 134, 1776-1778 (2009); doi: 10.1039/B906786H

[12] M. Bouvet, J. Simon, Electrical properties of rare earth bisphthalocyanines and bisnaphthalocyanines complexes, Chemical Physics Letters 172, 299-302 (1990); doi : 10.1016/00092614(90)85407-4

[13] Y. Chen, M. Bouvet, T. Sizun, G. Barochi, J. Rossignol, E. Lesniewska, Enhanced chemosensing of ammonia based on the novel molecular semiconductor-doped insulator (MSDI) heterojunctions, Sensors and Actuators B 155(1), 165-173 (2011); doi: 10.1016/j.snb.2010.11.042

[14] M. Bouvet, H. Xiong, V. Parra, Molecular semiconductor - doped insulator (MSDI) heterojunctions: Oligothiophene/bisphthalocyanine $\left(\mathrm{LuPC}_{2}\right)$ and perylene/bisphthalocyanine as new structures for gas sensing, Sensors and Actuators B, 145, 501-506 (2010); doi: 10.1016/j.snb.2009.12.064

[15] V. Parra, M. Rei Vilar, A. M. Botelho Rego, A. M. Ferraria, S. Boufi, M. L. Rodriguez-Mendez, E. Fonavs, I. Muzikante, M. Bouvet, Novel hybrid films based on cellulose/hydroxygallium phthalocyanine. Spectroscopic, surface and gas sensing studies, Langmuir, 23, 3712-3722 (2007); doi: 10.1021/la063114i

[16] M. Bouvet, A. Leroy, J. Simon, F. Tournilhac, G. Guillaud, P. Lessnick, A. Maillard, S. Spirkovitch, M. Debliquy, A. de Haan, A. Decroly, Detection and titration of ozone using metallophthalocyanine-based field-effect transistor, Sensors and Actuators $B, \quad 72, \quad 86-93 \quad$ (2001); doi: 10.1016/S0925-4005(00)00630-4

[17] M. Bouvet, G. Guillaud, A. Leroy, A. Maillard, S. Spirkovitch, F.-G. Tournilhac, Phthalocyaninebased field-effect transistor as ozone sensor, Sensors and Actuators B, 73, 63-70 (2001); doi: 10.1016/S0925-4005(00)00682-1

[18] M. Bouvet, Phthalocyanine - based field-effect transistors as gas sensors, Analytical and Bioanalytical Chemistry 384, 366-373 (2006); doi: 10.1007/s00216-005-3257-6

[19] T. Sizun, M. Bouvet, Y. Chen, J.-M. Suisse, G. Barochi, J. Rossignol, Differential study of substituted and unsubstituted cobalt phthalocyanines for gas sensor applications, Sensors and Actuators B 159, 163-170 (2011); doi: 10.1016/j.snb.2011.06.067

[20] G. Barochi, M. Bouvet, J. Rossignol, Development of microwave gas sensors, Sensors and Actuators B 157(2), 374-379 (2011); doi: 10.1016/j.snb.2011.04.059 